\title{
Development of an integrated model of care for allogeneic stem cell transplantation facilitated by eHealth — the SMILe study
}

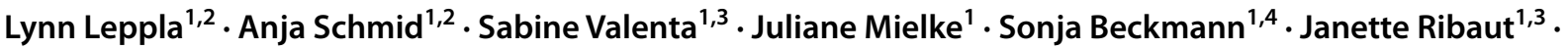 \\ Alexandra Teynor ${ }^{5} \cdot$ Fabienne Dobbels $^{6} \cdot$ Nathalie Duerinckx $^{6} \cdot$ Robert Zeiser $^{2} \cdot$ Monika Engelhardt $^{2} \cdot$ Sabine Gerull $^{3}$. \\ Sabina De Geest ${ }^{1,6,7} \cdot$ the SMILe study team
}

Received: 17 November 2020 / Accepted: 30 May 2021 / Published online: 5 July 2021

(c) The Author(s) 2021

\begin{abstract}
Purpose Allogeneic stem cell transplantation would benefit from re-engineering care towards an integrated eHealth-facilitated care model. With this paper we aim to: (1) describe the development of an integrated care model (ICM) in allogeneic SteM-cell-transplantatIon faciLitated by eHealth (SMILe) by combining implementation, behavioral, and computer science methods (e.g., contextual analysis, Behavior Change Wheel, and user-centered design combined with agile software development); and (2) describe that model's characteristics and its application in clinical practice.

Methods The SMILe intervention's development consisted of four steps, with implementation science methods informing each: (1) planning its set-up within a theoretical foundation; (2) using behavioral science methods to develop the content; (3) choosing and developing its delivery method (human/technology) using behavioral and computer science methods; and (4) describing its characteristics and application in clinical practice.

Results The SMILe intervention is embedded within the eHealth enhanced Chronic Care Model, entailing four self-management intervention modules, targeting monitoring and follow-up of important medical and symptom-related parameters, infection prevention, medication adherence, and physical activity. Interventions are delivered partly face-to-face by a care coordinator embedded within the transplant team, and partly via the SMILeApp that connects patients to the transplant team, who can monitor and rapidly respond to any relevant changes within 1 year post-transplant.

Conclusion This paper provides stepwise guidance on how implementation, behavioral, and computer science methods can be used to develop interventions aiming to improve care for stem cell transplant patients in real-world clinical settings. This new care model is currently being tested in a hybrid I effectiveness-implementation trial.
\end{abstract}

Keywords Allogeneic stem cell transplantation $\cdot$ eHealth $\cdot$ Integrated care $\cdot$ Implementation science $\cdot$ Behavioral science . User-centered design $\cdot$ Agile software development $\cdot$ Intervention development

Sabina De Geest

sabina.degeest@unibas.ch

1 Institute of Nursing Science, Department Public Health, University of Basel, Basel, Switzerland

2 Department of Medicine I, Faculty of Medicine, Medical Center University of Freiburg, Freiburg im Breisgau, Germany

3 Department of Hematology, University Hospital Basel, Basel, Switzerland

4 Center of Clinical Nursing Science, University Hospital Zurich, Zürich, Switzerland
5 Faculty of Computer Science, University of Applied Sciences Augsburg, Augsburg, Germany

6 Academic Centre for Nursing and Midwifery, Department of Public Health and Primary Care, KU Leuven, Leuven, Belgium

7 Nursing Science (INS), Department Public Health (DPH), Faculty of Medicine, University of Basel, Bernoullistrasse 28, CH-4056 Basel, Switzerland 


\section{Introduction}

Although allogeneic stem cell transplantation (alloSCT) recipients' survival has improved over recent years, significant risks remain for short- and long-term complications such as infections or graft-versus-host disease (GvHD) [1, 2]. Moreover, non-adherence to their therapeutic regimens is common and can negatively affect long-term outcomes [3-5]. Including psychosocial issues, alloSCT patients' comprehensive care needs demand self-management interventions embedded in an integrated care model (ICM) [6]. Based on chronic illness management principles, using multidisciplinary team-based approaches spanning settings and care levels [7], ICMs strengthen person-centered care, potentially improving medical, behavioral, and economic outcomes [8].

One excellent example is the Chronic Care Model (CCM). Its four inner dimensions-self-management support, decision support, clinical information systems, and delivery system design-guide the re-engineering of acute-care-oriented models towards chronic care principles [9]. The more are combined, the stronger the effect [10]. Considering healthcare's increasing digitalization, the updated eHealth enhanced Chronic Care Model (eCCM) explains how to strengthen all four dimensions via digitalization [11]. Systematic reviews and meta-analyses indicate that eHealth-facilitated ICMs improve biomedical, behavioral, psychosocial [12-14], and economic outcomes [13, 15]. In organ transplant recipients, these include improved medication adherence [16] and reduced re-hospitalizations [17]. In cancer patients, eHealth-facilitated care models integrating two or more eCCM dimensions led to, e.g., reduced symptom burden [18], re-hospitalizations, improved survival, quality of life $[19,20]$, and physical activity [21].

Regarding SCT care models, the only two RCTs focused respectively on one and two eCCM dimensions. For the first, Bryant et al. [22] implemented electronic patientreported outcomes into routine care during the first 2 weeks post-SCT ( $n=45$ autologous; $n=31$ allogeneic), followed by tailored self-management support leading to reduced peak symptom burden $(p=0.03)$. In the second, Syrjala et al. [23] found that for survivors $>3$ years postSCT ( $n=182$ autologous; $n=566$ allogeneic $)$, online selfmanagement and decision support led to reduced treatment distress $(p=0.032)$. However, both trials focused on very specific treatment phases; and neither was conceptually embedded in an ICM or comprehensively addressed multiple eCCM dimensions.

Moreover, implementing eHealth-facilitated ICMs into routine care is often problematic [24]. Adoption and sustainment problems commonly prevent eHealth applications' integration in established care models, with 44 to $67 \%$ of patients discontinuing the use [25-27]. Explanations include poor fit to context-dependent variables, deficits regarding behavioral effectiveness, and problems with the technology not meeting users' needs [28].

Combining implementation (e.g., contextual analysis), behavioral (e.g., behavior change theories), and computer science methods (e.g., agile software development, usercentered design) to develop eHealth-facilitated interventions could solve such problems [24, 28, 29]. Implementation science sets the long-range goal-sustainably improving the quality and effectiveness of patient care [30]. This means integrating methodological considerations such as stakeholder involvement, contextual analysis, and the choice and application of context-adapted implementation strategies [31]. Combined with a theory-guided content development using behavioral science and by developing the necessary technology around end-user needs and preferences using agile software development processes, maximize its usability and accessibility [28, 29, 32]. For eHealth component production, this combination promises fast, iteratively improved software versions that can be discussed regularly with end users (patients, clinicians) [33]. Following this formula, implementation/behavioral/computer science fusions should facilitate user-friendly, contextually targeted eHealth components ready to be embedded within ICMs.

Although alloSCT patients can clearly benefit from an eHealth-facilitated ICM, no such model currently exists nor has been prepared for implementation in real-world settings. Therefore, we are developing, implementing, and testing an allogeneic SteM-cell-transplantatIon faciLitated by $\mathbf{e H e a l t h}$ Integrated Care Model (SMILe-ICM) combining implementation and behavioral methods with computer science methods. This article first reports on the methods used to develop the SMILe-ICM, then describes its characteristics and application in clinical practice.

\section{Methods and results}

The development phase consisted of a sequence of four steps, with implementation science methods informing each (Fig. 1): (1) Choose the SMILe-ICM's theoretical foundation; (2) Develop four theory-guided self-management intervention modules; and (3) Choose and develop the intervention's delivery method(s), i.e., human and/or technology. With the SMILe-ICM's three-step development complete, the fourth step is to report on those steps, along with its characteristics and its application in daily clinical practice. All four steps are described below. 


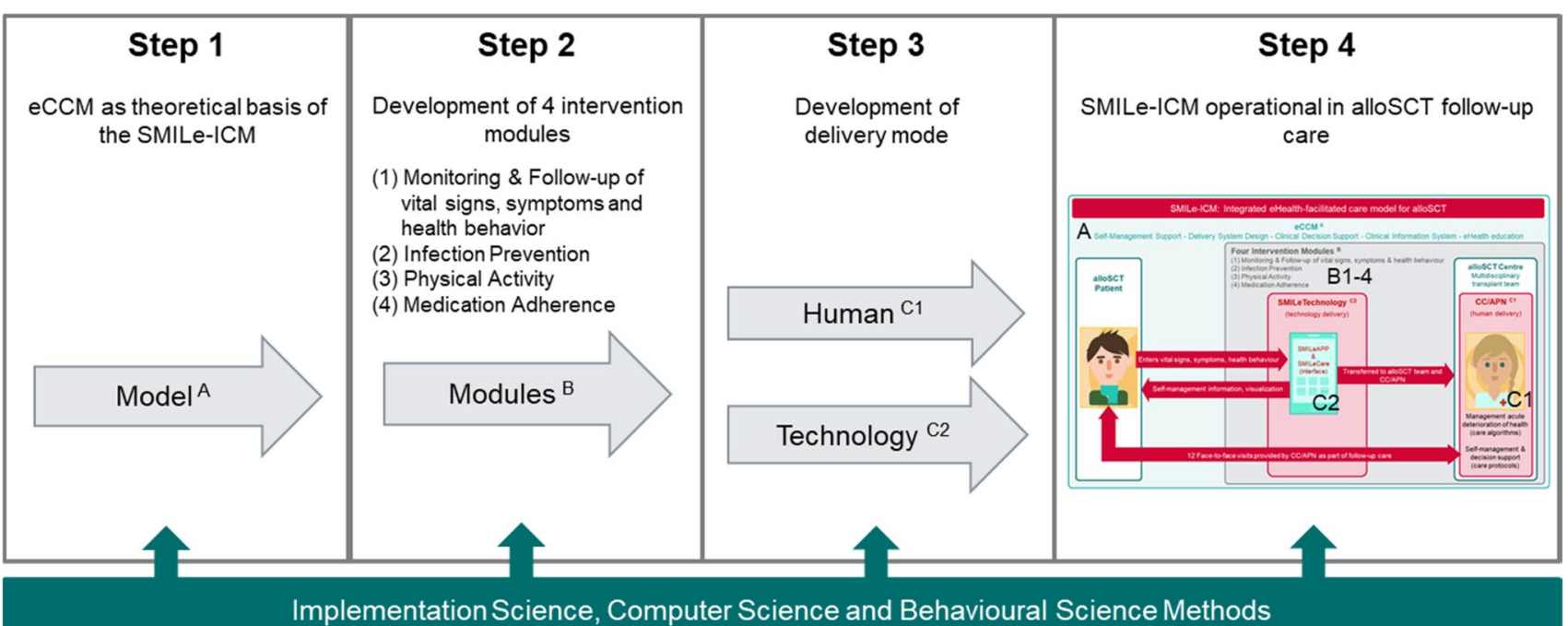

Fig. 1 The three subsequent steps of the development process building up to the SMILe-ICM described in step 4. Note: A, B, C also refer to Fig. 2 were the same elements can be found within the visualization of the SMILe integrated care model (ICM)

Step1: The SMILe-ICM's theoretical foundation

The SMILe-ICM is grounded in the eCCM (Fig. 1 step 1;

Fig. 2 A), a choice based on our contextual analysis, find- ings can be found in detail elsewhere [34]. Data from two surveys (60 patients/5 clinicians), three clinician focus groups and ten patient interviews indicated that the exist-

\section{SMILe-ICM: Integrated eHealth-facilitated care model for alloSCT}

$\operatorname{eCCM}^{\mathrm{A}}$

Self-Management Support - Delivery System Design - Clinical Decision Support - Clinical Information System - eHealth education

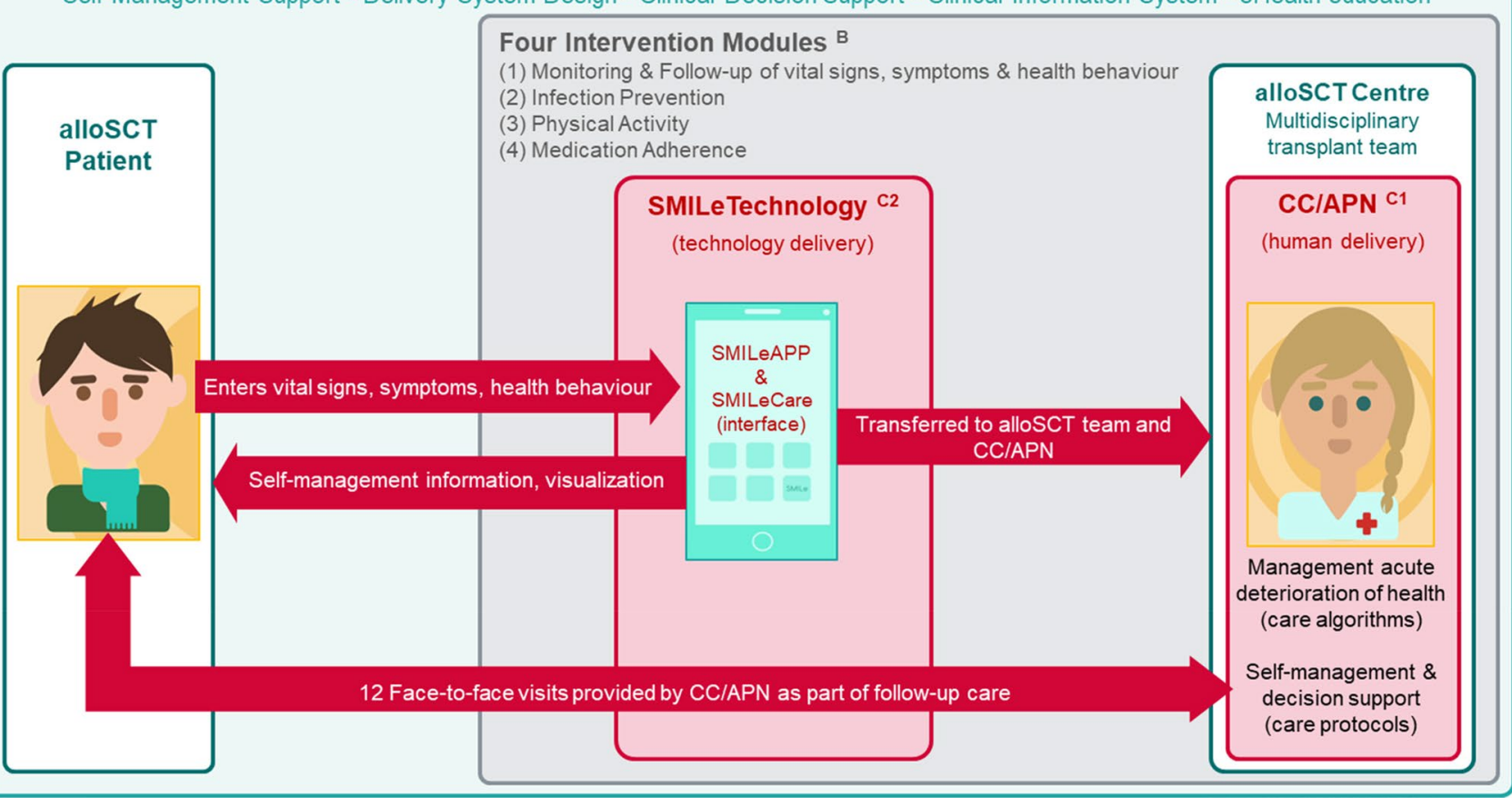

Fig. 2 The SMILe integrated care model. Note: $A=$ Five dimensions of the eHealth enhanced Chronic Care Model, $B=$ Four intervention modules delivered partly via human (alloSCT Transplant Team and
$C C=C 1)$ and partly via technology components $(=C 2) ; C C$, care coordinator; APN, advanced practice nurse 
ing care model is mainly acute care-driven: it focuses on diagnosing and curing patients; inpatient and outpatient care are separate, with limited collaboration between their clinician teams; outpatient alloSCT follow-up is primarily physician-centered, focusing mainly on medical aspects; no nurses are involved in care delivery and little attention is devoted to self-management support. Use of eCCM dimensions would quickly allow connections between inpatient and outpatient care teams by encouraging interdisciplinary teamwork. Furthermore, far from replacing human contact between patients and providers, eHealth should supplement that contact, helping maintain continuity of care and self-management support.

Step: 2 Theory-guided content development of the intervention modules

Our contextual analysis, empirical evidence, and clinicians' and patients' endorsements led us to produce four self-management intervention modules: monitoring and follow-up, infection prevention, medication adherence, and physical activity (Fig. 2 B). Content development followed the Behavior-Change Wheel (BCW). A widely used amalgamation of 19 behavior change theories [35], the BCW helps its users understand, explain, and change behaviors via stepwise development processes. With the Capability-Opportunity-Motivation-Behavior (COM-B) Model at its hub, the BCW meshes well with the Theoretical Domains Framework (TDF) [35], which includes 14 domains, synthesizing key theoretical constructs used in behavioral theories such as knowledge, skills, goals, or beliefs relating to the COM-B component.

For developing our intervention modules' content, we established an interdisciplinary team with expertise in implementation and behavioral science methods (nursing scientists, psychologist). Pairs of researchers conducted a literature search to identify empirical evidence regarding a specific self-management issue, its determinants, and possible target behaviors. Determinants were derived from empirical evidence, the contextual analysis [34], and clinical expertise of the team into the COM-B taxonomy, then discussed by the entire group. Where teams found multiple behaviors, the group chose which to target.

After choosing intervention functions for all targets, we selected appropriate TDF domains, behavioral change techniques (BCTs), and applied APEASE criteria (affordability, practicability, effectiveness, acceptability, safety, and equity) to each. Finally, the teams carefully reflected upon the mode of delivery (face-to-face and/ or technology-based) and added an additional step not covered by the $\mathrm{BCW}$. We wrote comprehensive protocols for the intervention segments' face-to-face visits and full descriptions of the functionalities to be digitized as user stories $[36,37]$.
User stories are commonly formulated in a role-featurereason format [38]. Each software functionality is presented as a sentence specifying the target user/role, the desired feature, and a reason/expected outcome, e.g., "As a patient/clinician, I want to monitor my pain intensity so that I can keep track of my pain trajectory." By supporting the translation of BCTs into software features, user stories inform the iterative software development process. Once the stories were articulated, they were transferred to the software development team, starting the digitalization process [36].

\section{Description of the four intervention modules}

To underpin our intervention modules' content, seven intervention functions reflecting all TDF domains and 42 different BCTs from Michie's taxonomy were chosen [35]. These informed 39 user stories. The following paragraphs describe the intervention modules (Fig. 2 B). Additional details for all modules regarding the target behaviors, behavioral issues, intervention components, user stories, behavior change techniques, intervention functions, TDF domains, and COM-B dimensions are presented in Supplementary Table 1.

Monitoring and follow-up This module targets alloSCT patients' insecurity regarding recognizing and reacting to new symptoms [34]. Our literature review and feedback cycles indicated that remote monitoring of patient-reported data effectively allows both accurate symptom recognition and timely reactions [18]. To support this behavior, we composed an initial set of 17 parameters for daily monitoring.

Parameter relevance was decided via an online survey of 12 German-speaking alloSCT experts. Using 6-point Likert-type scales $(0=$ not at all relevant $-5=$ extremely relevant), these experts were asked to rate the importance of symptoms covered by the PROVIVO alloSCT patientreported outcome questionnaire [39]. Inclusion of those with median ratings $\geq 4$ led to a selection of 12 from the original PROVIVO questionnaire, plus one more recommended by the group: signs of bleeding. The experts also added temperature, blood pressure, weight, and general wellbeing, resulting in 17 items to be monitored for this module. To support appropriate reactions, we collaborated closely with physicians involved in alloSCT care to define meaningful cut-off levels and feedback algorithms for each parameter (e.g., temperature $>38.5$ : contact the center immediately). Table 1 exemplarily summarizes the intervention modules' mechanisms for behavior change towards adequately recognizing, evaluating, and reacting to new symptoms. 


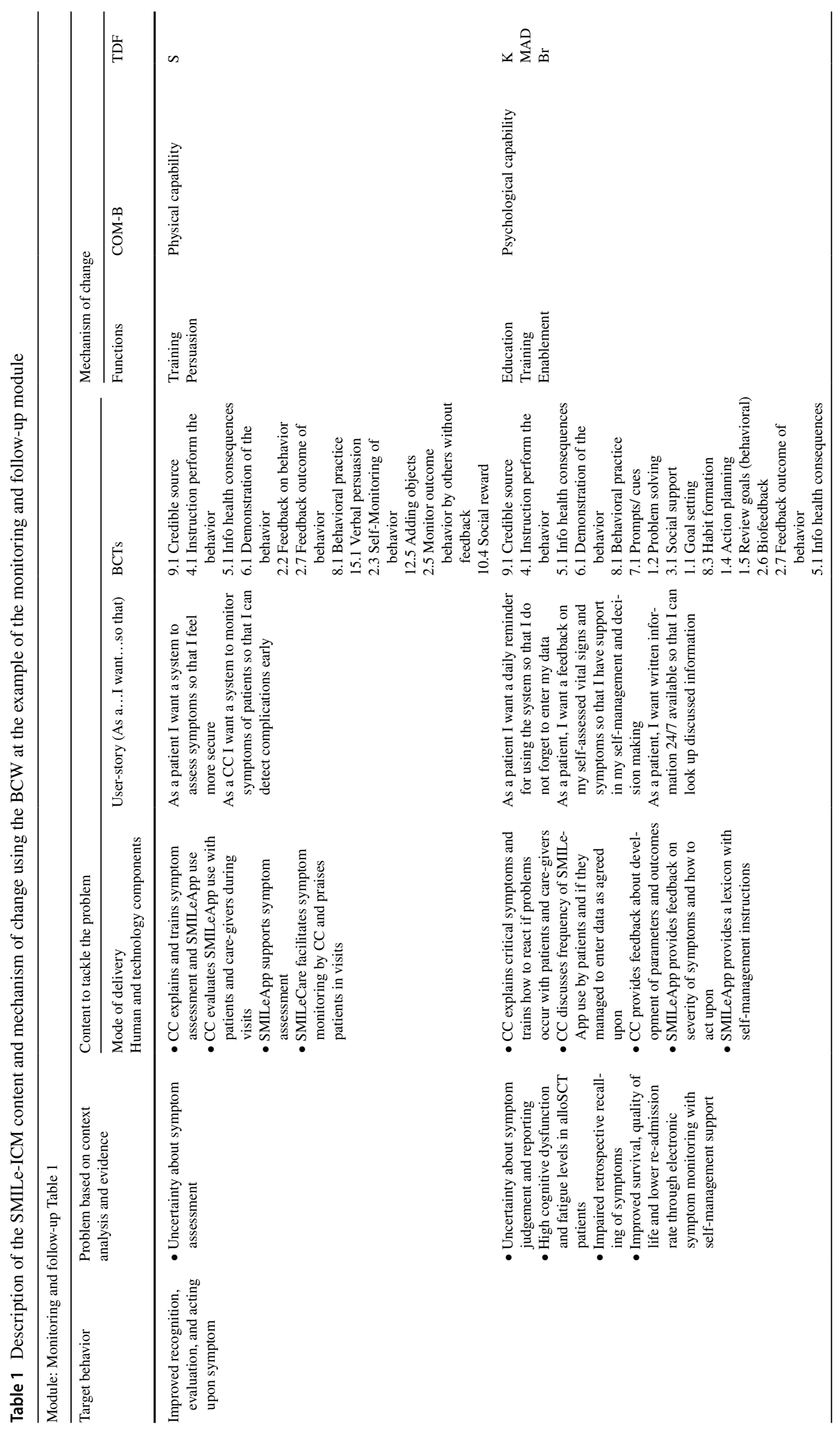




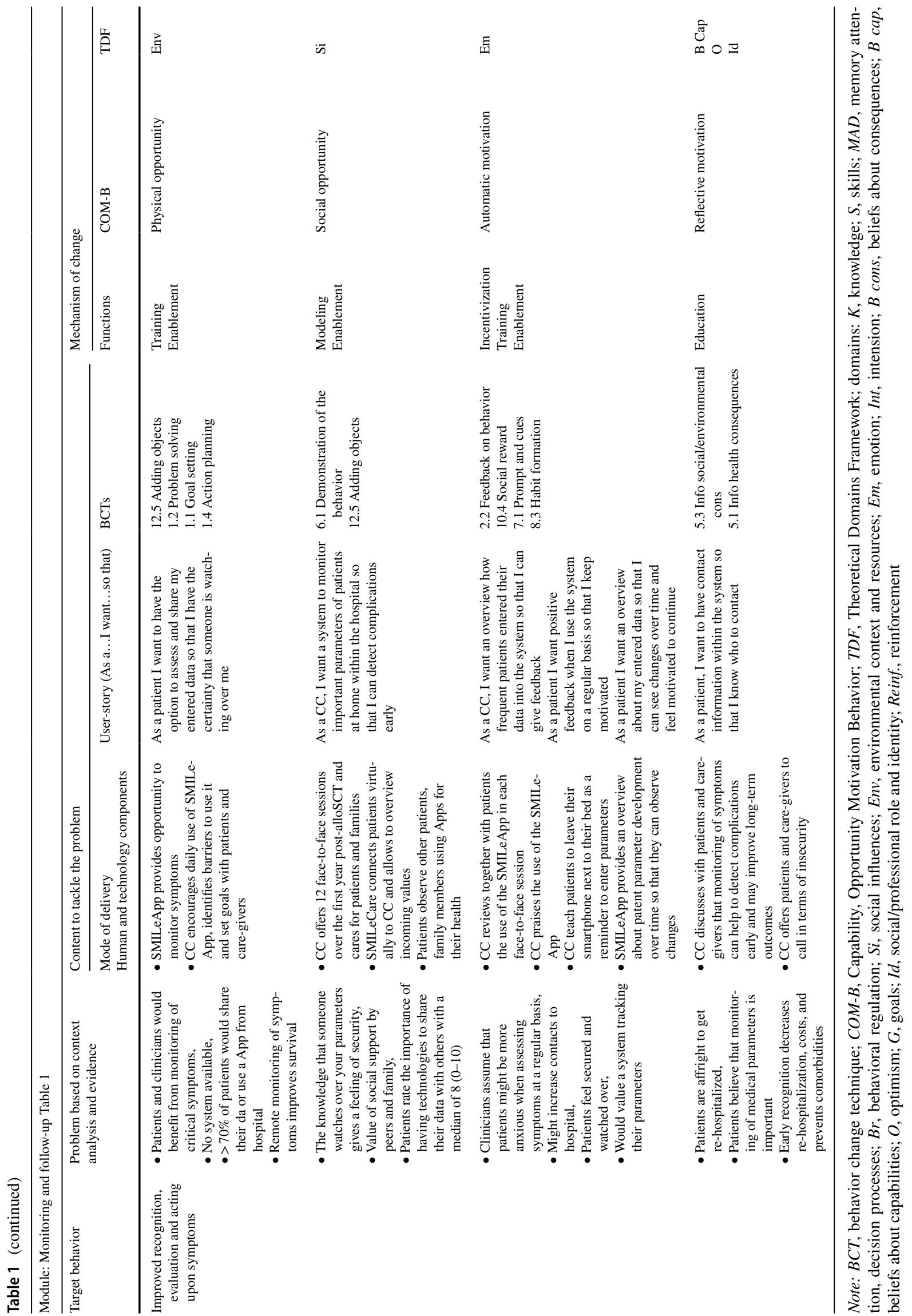


Table 2 The eCCM dimensions and described operationalization of the SMILe-ICM

\begin{tabular}{|c|c|c|c|}
\hline $\mathrm{eCCM}$ & $\begin{array}{l}\text { SMILe } \\
\text { technol- } \\
\text { ogy }\end{array}$ & $\mathrm{CC}$ & Operationalization \\
\hline \multirow[t]{4}{*}{ SM-S } & & $\mathrm{x}$ & $\begin{array}{l}\text { The CC provides patients with self-management support interventions beginning } 2 \text { weeks before until } 1 \text { year post- } \\
\text { alloSCT, delivering } 12 \text { face-to-face sessions covering four modules }\end{array}$ \\
\hline & $\mathrm{x}$ & & Patients receive algorithm powered feedback based on entered parameters via the SMILeApp \\
\hline & $\mathrm{x}$ & $\mathrm{x}$ & SMILeCare allows to detect complications early and allows to provide tailored additional face-to-face session \\
\hline & & $\mathrm{x}$ & In case of highly burdened patients, $\mathrm{CC}$ provides additional support and/or case-management \\
\hline \multirow[t]{4}{*}{ DSD } & $\mathrm{x}$ & & $\begin{array}{l}\text { The use of information technology (SMILeApp and SMILeCare) is a new element and allows to adapt care-processes } \\
\text { with the goal of optimizing both resource use and clinical outcomes }\end{array}$ \\
\hline & & $\mathrm{x}$ & Advanced practice oncology nurses need to be in place to work in the new role of a CC \\
\hline & & $\mathrm{x}$ & $\begin{array}{l}\text { The introduction of the CC is a new element in alloSCT follow-up. Accordingly, care processes need to be adapted by } \\
\text { the alloSCT center and weekly interdisciplinary discussion rounds should be implemented }\end{array}$ \\
\hline & $\mathrm{x}$ & $\mathrm{x}$ & The SMILeApp contains and CC uses developed educational materials for each module \\
\hline \multirow[t]{3}{*}{ CDS } & $\mathrm{x}$ & & $\begin{array}{l}\text { If serious symptoms are entered patients receive algorithm-based feedback how fast they should contact the transplant } \\
\text { center }\end{array}$ \\
\hline & & $\mathrm{x}$ & $\begin{array}{l}\text { The CC's work is guided by protocols that build on the alloSCT centers' clinical practice pattern guidelines and have } \\
\text { been approved by the centers' physicians }\end{array}$ \\
\hline & & $\mathrm{x}$ & $\begin{array}{l}\text { The CC can discuss treatment decisions/changes pro-actively with attending physicians when necessary based on the } \\
\text { monitoring }\end{array}$ \\
\hline \multirow[t]{2}{*}{ CIS } & $\mathrm{x}$ & & $\begin{array}{l}\text { Vital signs, symptoms, and health behaviors of home dwelling alloSCT patients are captured by the SMILeApp and } \\
\text { transferred to the hospital where the CC can overview them }\end{array}$ \\
\hline & $\mathrm{x}$ & $\mathrm{x}$ & The CC can access the patient data if agreed to by the patient and share it with the attending physician if needed \\
\hline eHed & & $\mathrm{x}$ & The patients and the CC are trained to work respectively with the SMILeApp and SMILeCare applications \\
\hline
\end{tabular}

Note: eCCM, eHealth enhanced Chronic Care Model; $C C$, care coordinator; $S M$-S, self-management support; $D S D$, delivery system design; $C D S$, clinical decision support; $C I S$, clinical information system; $e H e d$, eHealth education

Infection prevention This module targets patients' challenges regarding infection prevention measures, often leading to the significant burden of infection-related re-hospitalization-most common in the first 2 years post-alloSCT [2, 34]. Three target behaviors are covered: (1) adequate hand hygiene; (2) airborne pathogen-related risk reduction; and (3) safe food handling, preparation, and consumption. This module's content required adaptivity, depending on participants' time since transplantation and immunity status. Patients' severely immuno-compromised or presenting signs of GvHD need stricter recommendations; for those with more stable immune systems, they can be loosened. This module also adds one monitoring parameter: adherence to infection prevention measures.

Medication adherence This module responds to patients' calls for immunosuppressant intake support [34]. More than $50 \%$ of alloSCT patients reported immunosuppressant nonadherence in view of errors in correct taking and timing [3-5]. Immunosuppressant medication non-adherence has been linked to GvHD, which frequently leads to poor clinical outcomes [3, 4, 40]. Therefore, the Medication Adherence module targets the implementation dimension of medication adherence (taking and timing behavior). This module also adds one monitoring parameter: medication intake. A detailed description of this module's development process has been published elsewhere [37] and is a blueprint for how other modules were developed.

Physical activity This module targets alloSCT patients' commonly reduced physical capability due to pre-transplant treatment or post-transplant complications. In addition to lowering patients' quality of life, these can shorten survival [41]. Conversely, improved physical condition is linked to improved health outcomes [34, 41]. Our contextual analysis indicated a need for support of physical activity before, during, and post-alloSCT.

However, review of all available evidence indicated that improving physical activity would be overly ambitious as a target behavior: patients are too weak in the first month's post-alloSCT. Therefore, we reformulated the target behavior to "reducing sedentary bouts," increasing patients' physical activity alongside their energy levels. While we chose daily step count as an indirect measure of inactivity, this obviously also indicates physical activity. All patients receive step counters and training on how and when to wear them. This module adds daily step count as a monitoring parameter. 


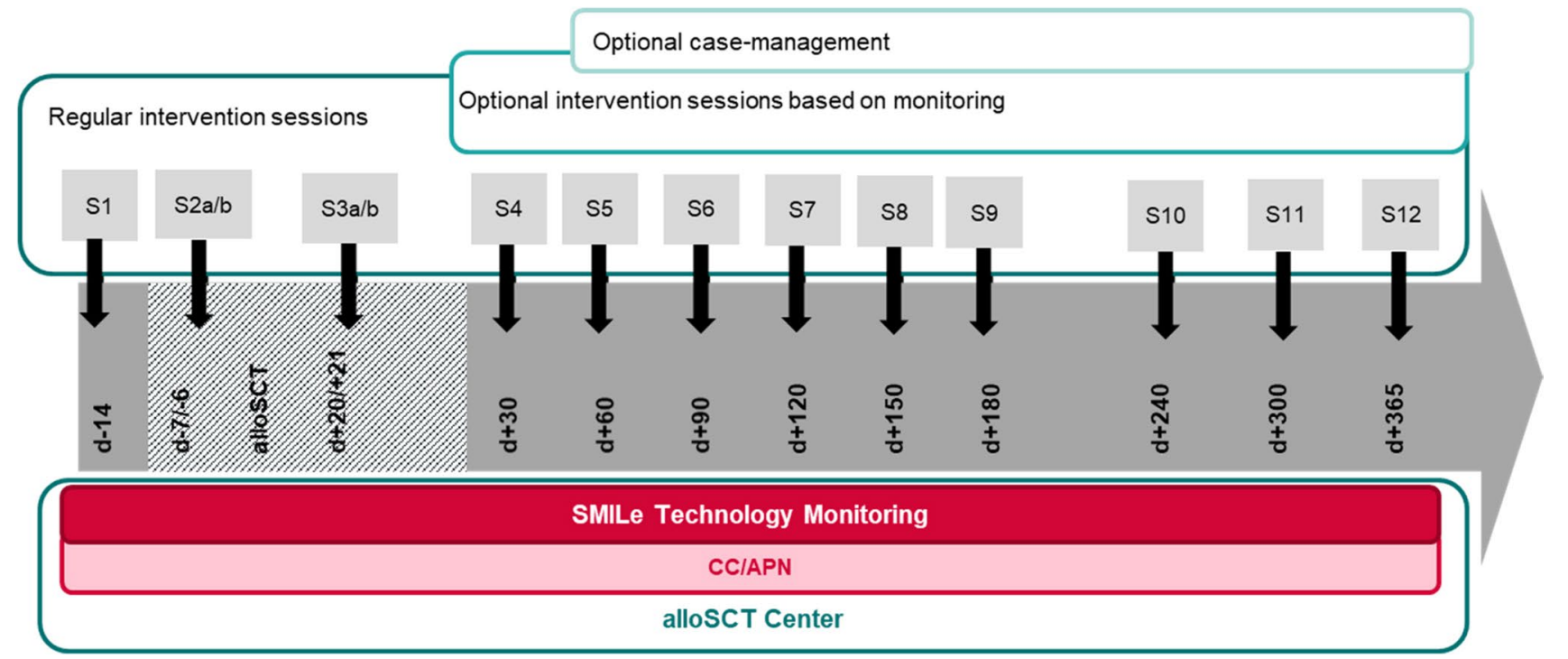

Fig. 3 Intervention timing and dosage within the SMILe integrated care model

Step: 3 Choice and development of intervention delivery methods

Both patients and clinicians preferred a combined face-toface and eHealth-enhanced intervention [34]. We operationalized all four eCCM dimensions (Table 2) and determined the most effective delivery methods (human/technology).

\section{The human-delivered components}

The SMILe-ICM's human-delivered components are 12 face-to-face sessions with an advanced practice nurse (APN)/care coordinator (CC, delivery system design; selfmanagement support). Embedded in the alloSCT team, in addition to coordinating patient care and delivering information and training, the $\mathrm{CC}$ provides the desired human factor. Additionally, working closely with the inpatient and outpatient teams, the CC can strengthen the links between the two.

Patients are monitored closely during their inpatient stay, but need to build post-discharge health self-management skills. Immediately post-discharge, they return 4-8 times per month as necessary for follow-up. As their conditions stabilize, follow-up intervals gradually increase to once yearly. As the patients' first point of contact, the CC delivers all required self-management interventions within (self-management support, delivery system design). All face-to-face sessions will follow a detailed intervention protocol, which guides the intervention delivery and support fidelity among the CCs while delivering the intervention modules. In addition to face-to-face contacts, the $\mathrm{CC}$ will also be connected with the patients via the SMILe technology enabling rapid responses to early signs of health deterioration.

\section{The technology components}

The SMILe technology includes the SMILeApp for the patient and the browser-based SMILeCare monitoring component for the care team (delivery system design; clinical information systems). Via the SMILeApp, patients are encouraged to daily enter data for 20 monitoring parameters. These include ratings of general well-being, temperature, weight, and blood pressure measurements, 13 symptomrelated parameters (pain; signs of bleeding; nausea; emesis; diarrhea; skin rash; mouth or throat sores; shortness of breath; cough; pain or burning at urination; fatigue, tiredness, or lack of energy; difficulty swallowing; decreased appetite), and three behavioral measures (adherence to infection prevention measures and medication intake, number of steps). According to the data patients enter, they receive automated feedback from the SMILeApp concerning selfmanagement or necessary actions (self-management support; decision support). If one or more parameters exceed pre-defined cut-offs, they receive instructions to contact the transplant center within the next 2 days, as soon as convenient, immediately, or even to call an ambulance immediately.

Furthermore, patients have access to self-management and behavioral support information via a lexicon of complications and parameters assessed by the SMILeApp (selfmanagement support). With the patient's consent, the data entered are transferred to the university hospital data center, where the $\mathrm{CC}$ reviews incoming values and visualizations of 
their development via the SMILeCare monitoring software (clinical information system). The $\mathrm{CC}$ reacts following the SMILe risk-adjusted care protocol (decision support, clinical information systems, delivery system design). Based on the same algorithms that guide the CC, other alloSCT team members will be involved as appropriate. Both patients and CCs receive special SMILe technology training (eHealth education).

During the development phase, the SMILeApp was subjected to two rounds of classical user tests involving 5-6 patients per round, resulting in high user experience ratings [36]. To help the development team clarify design questions (e.g., the logo), and to discuss certain functionalities' acceptability (e.g., frequency data entry), we also retrieved patient and $\mathrm{CC}$ feedback.

Step: 4 SMILe intervention characteristics and application in daily clinical practice

The three above-described steps resulted in the operational SMILe-ICM's intervention characteristics ready for roll-out in clinical practice (Fig. 2). The following section describes the intervention's application in daily clinical practice.

To recruit patients, the $\mathrm{CC}$ contacts patients as soon as they are listed for transplantation (1 to 5 weeks pre-admission), to schedule their first face-to-face session. Patients support needs are highest in the first 6 months post-discharge. Therefore, the $\mathrm{CC}$ delivers most interventions between days -10 pre- and +180 post-alloSCT in close collaboration with the alloSCT team. Patient contact begins before the inpatient stay and continues via scheduled outpatient clinic appointments as part of usual follow-up care. The $\mathrm{CC}$ delivers the highest-frequency intervention dosage over the first 2 months. This usually drops in months 3-6, with the lowest dosage occurring in month's 7-12 post-alloSCT (Fig. 3). The CC uses the face-to-face sessions to deliver/ reiterate oral instructions and/or tailored self-management and behavioral support. Already in the first session prealloSCT, patients receive a step counter and the SMILeApp, either installed on their smartphone or on a hospital-provided tablet computer. After teaching them to use the step counter, the CC trains them to enter symptoms (e.g., skin rash) to the app, how to interpret the app's feedback, and how to react if no feedback is received. While hospitalized, they receive as many SMILeApp training sessions as necessary to use it confidently. Before discharge, to develop the habit of entering their daily data, patients also practice this until proficient.

Depending on the patient's condition, the intervention protocol allows stepping up of intervention dosage, i.e., for those reporting parameters above cut-off levels or requiring additional support, the $\mathrm{CC}$ will immediately contact the responsible clinical team (e.g., treating physician) in the inpatient or outpatient setting based on predefined protocols. Where patients have two or more uncontrolled symptoms, the $\mathrm{CC}$ also provides case management (Fig. 3).

\section{Discussion}

This is the first paper to report on the theory-driven, evidence-based development of an eHealth-facilitated alloSCT ICM combining implementation, behavioral, and computer science methods. This includes, respectively, a contextual analysis, the Behavior Change Wheel, and user-centered design informing the agile software development. It provides not only information on this complex intervention's content, but also step-by-step guidance on how to develop a similar care model for any context and real-world implementation.

Traditional models of care in alloSCT settings are predominantly acute care-driven, with limited focus on selfmanagement. Based on prevailing evidence and insights from our contextual analysis, a re-engineering of alloSCT care towards an integrated chronic illness approach is urgently needed. In terms of care coordination and selfmanagement requirements, the first months post-alloSCT are the most complex. These involve high re-hospitalization risks due to various complications - the most frequent being infections and GvHD [2, 6]. EHealth-facilitated ICMs offer promising methods of improving outcomes across a variety of chronic illnesses. However, few have been applied in alloSCT settings, and none has focused on inpatients' transitions to home-a moment when continuity of care is challenged and complications frequently arise [42]. Such findings are consistent with those of our contextual analysis, which indicated that patients' greatest support needs occur during the first year post-alloSCT [34].

Numerous commercial eHealth applications are available for cancer settings, some of which are connected to the patients care teams. However, while a small number are either embedded within an ICM approach or are theorybased [18, 28], to our knowledge, these applications have not been developed based on an implementation science approach to ease the adaption, implementation, and sustainability in real-world settings. Consequently, these interventions frequently function as black boxes regarding development, symptoms monitored, or mechanisms underpinning their target behavioral changes. For example, a critical review of 23 eHealth apps for patients with cancer found that no theoretical basis was generally present: only six of the 23 even referred theories or behavior change models [28]. Of the other available offerings, fewer than $20 \%$ refer to empirical studies or background source information; only $11.3 \%$ are evidence-based; and just under $10.3 \%$ involve clinicians in their development processes [43-45]. And while many 
report user-centered design approaches, few support such claims with insights into end-user involvement or contextspecific adaptions. This might result from a strong focus on technology development, with less attention to theoretical underpinnings: such an approach may appear to shorten the path to implementation. In addition, while many applications pay some attention to context-specific requirements, the broader perspective-re-engineering entire care teams and processes-is missing entirely. Overall, alongside the general lack of theoretical underpinnings or contextspecific adaptions, the non-use or non-transparent use of user-centered design methodology precludes the uptake and sustained clinical use of virtually any off-the-rack eHealth application in real-world settings.

Furthermore, both Zhao et al. (2016) and Hamel et al. (2019) argue that improving the effectiveness of eHealth design demands the ongoing involvement of all relevant stakeholders to produce a thorough understanding of enduser needs and preferences, with the most effective applications supplying real-time feedback, individualizable elements, and evidence-based medical information [28, 45]. This reflects the findings of our contextual analysis, which revealed that our target patients and clinicians are not interested in a stand-alone eHealth solution, but in a purposedesigned combination of human- and technology-delivered components best reflected by an eHealth-facilitated ICM [34]. It also buttresses the argument that embedding eHealth components in integrated care models facilitates muchneeded continuity of care and self-management support between outpatient clinic visits [42].

Our development process included certain notable challenges. Most importantly, implementing an eHealth-facilitated ICM is more than adding an intervention. It is about re-engineering care processes. Within transplant centers, a systematic implementation requires personnel, time-related, and financial resources. Total resource use and associated costs cover all phases, from initial development/adaption to roll out in clinical practice. Indeed, the initial development of the first SMILe-ICM version-starting with a contextual analysis, followed by the content, and software development (within an interdisciplinary team conversant with three methodologies)—-took us 2 years [36, 37].

This was time well invested. It allowed us to lay the foundation for a product that fits both the intended context and the target users' requirements and we believe that this maximizes the likelihood of success when rolling-out and sustaining the intervention in real clinical practice. The preparation of the roll out in clinical practice required team meetings, development/adaption of educational materials, and to secure server space. To operate the SMILe-ICM in clinical practice, we factored in a $100-150 \%$ full-time care coordinator position, plus yearly server and software maintenance costs. Specific cost details are context specific. Our currently running hybrid-1 effectiveness-implementation RCT of the first SMILe-ICM will be followed by a full economic analysis. This will include the patients' medical resource use over a 1-year follow-up and will be weighed against those costs of operating the SMILe-ICM intervention.

The SMILe-ICM trial's full results will provide further clinical and implementation outcomes for a first center in Germany. We have already adapted it to a Swiss center, where we are currently preparing for the adapted version's implementation and testing phase. Even after the final evaluation, this will allow continuing insights into adaption costs.

Limitations of our human and time resources prevented the completion of the digitalization process as originally planned. A serious regulatory barrier was the main reason for this: The European Medical Device Regulation (MDR; EU2017/745) defines the originally planned SMILeApp as a class $2 b$ medical device, necessitating, e.g., tightly regulated development, certification, and ongoing quality management. Unable to meet these requirements, our academic project team was forced to cut back the planned level of digitization. While the monitoring and follow-up module is now digitalized with intermitted automated feedback and decision support, patients receive these information's as written leaflets. The other modules are still purely in a face-to-face delivering mode. In parallel of testing the SMILe-ICM, following all regulatory requirements (MDR; EU2017/745), the digitalization process of the eHealth components facilitating the face-to-face components will continue until all module components are digitalized and compliant with class $2 \mathrm{~b}$ medical device regulations. However, testing the system at an early stage provides insights influencing the creation of future modules; and we believe that the methods used increase the probability of sustainable implementation and acceptance in real-world clinical practice, while reducing research resource waste.

\section{Conclusions}

We found that the alloSCT setting would benefit strongly from the re-engineering of its care teams and processes towards an eHealth-facilitated ICM. Despite high levels of activity in the eHealth sector, though, empirical evidence is scarce. Many available eHealth applications are neither embedded within an ICM nor offer information regarding their theoretical underpinnings nor explanations of how contextual factors, end-user involvement, or 
effective behavior change techniques were integrated into their development processes. With this report, in addition to providing step-by-step guidance for development of an eHealth-facilitated ICM, we describe how the resulting model can be integrated within alloSCT patient care. We developed the SMILe-ICM via an iterative process merging implementation, behavioral, and computer science methods. This combination facilitates the development and tailoring of meaningful theory- and evidence-based interventions to end users' context-specific needs and preferences, thereby giving the resulting interventions the highest possible chance of uptake and sustained use.

Supplementary Information The online version contains supplementary material available at https://doi.org/10.1007/s00520-021-06328-0.

Acknowledgements We would like to thank all the involved patients, caregivers, and clinicians from the participating transplant centers. Furthermore, we thank Chris Shultis for editing the manuscript. SMILe Study Team in alphabetical order: Sonja Beckmann, Dora Bolliger, Yves Chalandon, Sabina De Geest, Sabine Degen, Fabienne Dobbels, Nathalie Duerinckx, Monika Engelhardt, Margerita Fürmann, Sabine Gerull, Florian Grossmann, Monika Hasemann, Philipp Heidegger, Anja Hermann, Sandra Hobelsberger, Mylen Husel, Katharina Koehly, Marina Lemcke, Lynn Leppla, Birgit Maier, AnneClaire Mamez, Stavoula Masouridi, Juliane Mielke, Gyathri Nair, Daniela Neupert, Jakob Passweg, Stefan Pschenitza, Sigrun Reitwiessner, Jannette Ribaut, Dennis Rockstein, Urs Schanz, Anja Schmid, Helen Schoemans, Tobias Schulz, Vanessa Schumacher, Yulia Senft, Alexandra Teynor, Sabine Valenta, Viktor Werlitz, Verena WitzigBrändli, Robert Zeiser.

Author contribution The manuscript was written by following the CONSORT eHealth and TIDieR checklist for intervention description. S. De Geest, L. Leppla, A. Teynor, and S. Valenta contributed to the study conception and design. Material preparation, data collection, and analysis were performed by L. Leppla, A. Schmid, S. Valenta, J. Mielke, S. Beckmann, J. Ribaut, F. Dobbels, N. Duerinckx, and S. De Geest. Input, revisions, and supervision: S. De Geest, M. Engelhardt, R. Zeiser, A. Teynor, S. Gerull. The first draft of the manuscript was written by L. Leppla and all authors commented and revised subsequent versions. All authors read and approved the final manuscript.

Funding Open Access funding provided by Universität Basel (Universitätsbibliothek Basel). The study was funded by the German B. Braun Stiftung (Grant BBST-D-17-00077) and the Swiss Cancer League (Grant HSR-4362-11-2017).

\section{Data Availability N/A}

Code availability The SMILe technology is still in a testing and developing face; software codes cannot be made available yet.

\section{Declarations}

Ethics approval Approval was granted by the local ethics committee of the University of Freiburg im Breisgau, German (EK67/17).

Consent to participate Informed consent was obtained from all individual participants included in the study.

\section{Consent to publication N/A.}

Conflict of interest The authors declare no competing interests.

Open Access This article is licensed under a Creative Commons Attribution 4.0 International License, which permits use, sharing, adaptation, distribution and reproduction in any medium or format, as long as you give appropriate credit to the original author(s) and the source, provide a link to the Creative Commons licence, and indicate if changes were made. The images or other third party material in this article are included in the article's Creative Commons licence, unless indicated otherwise in a credit line to the material. If material is not included in the article's Creative Commons licence and your intended use is not permitted by statutory regulation or exceeds the permitted use, you will need to obtain permission directly from the copyright holder. To view a copy of this licence, visit http://creativecommons.org/licenses/by/4.0/.

\section{References}

1. Gifford G, Sim J, Horne A, Ma D (2014) Health status, late effects and long-term survivorship of allogeneic bone marrow transplantation: a retrospective study. Int Med J 44(2):139-147

2. Chow EJ, Cushing-Haugen KL, Cheng GS, Boeckh M, Khera N, Lee SJ, Leisenring WM, Martin PJ, Mueller BA, Schwartz SM, Baker KS (2017) Morbidity and mortality differences between hematopoietic cell transplantation survivors and other cancer survivors. J Clin Oncol 35(3):306-313. https://doi.org/10.1200/jco. 2016.68.8457

3. Gresch B, Kirsch M, Fierz K, Halter JP, Nair G, Denhaerynck K, De Geest $S$ (2017) Medication nonadherence to immunosuppressants after adult allogeneic haematopoietic stem cell transplantation: a multicentre cross-sectional study. Bone Marrow Transplant 52(2):304-306. https://doi.org/10.1038/bmt.2016.262

4. Ice LL, Bartoo GT, McCullough KB, Wolf RC, Dierkhising RA, Mara KC, Jowsey-Gregoire SG, Damlaj M, Litzow MR, Merten JA (2020) A prospective survey of outpatient medication adherence in adult allogeneic hematopoietic stem cell transplant patients. Biol Blood Marrow Transplant

5. Kirsch M, Götz A, Halter J, Schanz U, Stussi G, Dobbels F, De Geest S (2014) Differences in health behaviour between recipients of allogeneic haematopoietic SCT and the general population: a matched control study. Bone MarrowTransplant 49(9):1223-1230

6. Majhail NS (2017) Long-term complications after hematopoietic cell transplantation. Hematol Oncol Stem Cell Ther

7. Goodwin N (2016) Understanding integrated care. Int J Integr Care 16 (4)

8. Nuño R, Coleman K, Bengoa R, Sauto R (2012) Integrated care for chronic conditions: the contribution of the ICCC Framework. Health Policy 105(1):55-64

9. Wagner Q EH, Austin BT, Von Korff M (1996) Organizing care for patients with chronic illness. Milbank:511-544

10. Bodenheimer T, Wagner EH, Grumbach K (2002) Improving primary care for patients with chronic illnessthe chronic care model, Part 2. JAMA 288(15):1909-1914. https://doi.org/10.1001/jama. 288.15.1909

11. Gee PM, Greenwood DA, Paterniti DA, Ward D, Miller LMS (2015) The eHealth enhanced chronic care model: a theory derivation approach. J Med Internet Res 17 (4)

12. Kuijpers W, Groen WG, Aaronson NK, van Harten WH (2013) A systematic review of web-based interventions for patient empowerment and physical activity in chronic diseases: relevance for 
cancer survivors. J Med Internet Res 15(2):e37. https://doi.org/ 10.2196/jmir.2281

13. Elbert NJ, van Os-Medendorp H, van Renselaar W, Ekeland AG, Hakkaart-van Roijen L, Raat H, Nijsten TE, Pasmans SG (2014) Effectiveness and cost-effectiveness of ehealth interventions in somatic diseases: a systematic review of systematic reviews and meta-analyses. J Med Int Res 16 (4)

14. Widmer RJ, Collins NM, Collins CS, West CP, Lerman LO, Lerman A Digital health interventions for the prevention of cardiovascular disease: a systematic review and meta-analysis. In: Mayo Clin Proc, 2015. vol 4. Elsevier, pp 469-480

15. Michaud TL, Zhou J, McCarthy MA, Siahpush M, Su D (2018) Costs of home-based telemedicine programs: a systematic review. Int J Technol Assess Health Care 34(4):410-418

16. Tang J, James L, Howell M, Tong A, Wong G (2020) eHealth interventions for solid organ transplant recipients: a systematic review and meta-analysis of randomized controlled trials. Transplantation. https://doi.org/10.1097/tp.0000000000003294

17. Kaier K, Hils S, Fetzer S, Hehn P, Schmid A, Hauschke D, Bogatyreva L, Jänigen B, Pisarski P (2017) Results of a randomized controlled trial analyzing telemedically supported case management in the first year after living donor kidney transplantation-a budget impact analysis from the healthcare perspective. Health Econ Rev 7(1):1

18. Aapro M, Bossi P, Dasari A, Fallowfield L, Gascón P, Geller M, Jordan K, Kim J, Martin K, Porzig S (2020) Digital health for optimal supportive care in oncology: benefits, limits, and future perspectives. Support Care Cancer. https://doi.org/10.1007/ s00520-020-05539-1

19. Basch E, Deal AM, Dueck AC, Scher HI, Kris MG, Hudis C, Schrag D (2017) Overall survival results of a trial assessing patient-reported outcomes for symptom monitoring during routine cancer treatment. Jama

20. Basch E, Deal AM, Kris MG, Scher HI, Hudis CA, Sabbatini P, Rogak L, Bennett AV, Dueck AC, Atkinson TM (2015) Symptom monitoring with patient-reported outcomes during routine cancer treatment: a randomized controlled trial. J Clin Oncol :JCO630830

21. Haberlin C, O'Dwyer T, Mockler D, Moran J, O'Donnell DM, Broderick J (2018) The use of eHealth to promote physical activity in cancer survivors: a systematic review. Support Care Cancer:1-14

22. Bryant AL, Coffman E, Phillips B, Tan X, Bullard E, Hirschey R, Bradley J, Bennett AV, Stover AM, Song L (2020) Pilot randomized trial of an electronic symptom monitoring and reporting intervention for hospitalized adults undergoing hematopoietic stem cell transplantation. Support Care Cancer 28(3):1223-1231

23. Syrjala KL, Jean CY, Artherholt SB, Romano JM, Crouch M-L, Fiscalini AS, Hegel MT, Flowers ME, Martin PJ, Leisenring WM (2018) An online randomized controlled trial, with or without problem-solving treatment, for long-term cancer survivors after hematopoietic cell transplantation. J Cancer Survivorship 12(4):560-570

24. Glasgow RE, Phillips SM, Sanchez MA (2014) Implementation science approaches for integrating eHealth research into practice and policy. Int J Med Inform 83(7):e1-e11

25. Jeffs E, Vollam S, Young JD, Horsington L, Lynch B, Watkinson PJ (2016) Wearable monitors for patients following discharge from an intensive care unit: practical lessons learnt from an observational study. J Adv Nursing 72(8):1851-1862

26. Thies K, Anderson D, Cramer B (2017) Lack of adoption of a mobile app to support patient self-management of diabetes and hypertension in a federally qualified health center: interview analysis of staff and patients in a failed randomized trial. JMIR Hum Factors 4(4):e24. https://doi.org/10.2196/humanfactors.7709
27. Simblett S, Greer B, Matcham F, Curtis H, Polhemus A, Ferrão J, Gamble P, Wykes T (2018) Barriers to and facilitators of engagement with remote measurement technology for managing health: systematic review and content analysis of findings. J Med Int Res20 (7)

28. Hamel LM, Thompson HS, Albrecht TL, Harper FW (2019) Designing and testing apps to support patients with cancer: looking to behavioral science to lead the way. JMIR Cancer 5 (1):e12317

29. Dopp AR, Parisi KE, Munson SA, Lyon AR (2020) Aligning implementation and user-centered design strategies to enhance the impact of health services: results from a concept mapping study. Implement Sci Commun 1:17. https://doi.org/10.1186/ s43058-020-00020-w

30. Eccles MP, Mittman BS (2006) Welcome to implementation science. Implement Sci 1(1):1. https://doi.org/10.1186/ 1748-5908-1-1

31. Peters DH, Adam T, Alonge O, Agyepong IA, Tran N (2013) Implementation research: what it is and how to do it. BMJ 347:f6753

32. Larusdottir M, Gulliksen J, Cajander $\AA$ (2017) A license to kill-Improving UCSD in Agile development. J Syst Software 123:214-222

33. Schwaber K, Beedle M (2002) gilè software development with scrum.

34. Leppla L, Mielke J, Kunze M, Mauthner O, Teynor A, Valenta S, Vanhoof J, Dobbels F, Berben L, Zeiser R (2020) Clinicians and patients perspectives on follow-up care and eHealth support after allogeneic hematopoietic stem cell transplantation: a mixedmethods contextual analysis as part of the SMILe study. European J Oncol Nurs 45:101723. https://doi.org/10.1016/j.ejon.2020. 101723

35. Michie S, Atkins L, West R, (2014) The behaviour change wheel: a guide to designing interventions. Silverback Publishing, Surrey. ISBN-13

36. Leppla L, Hobelsberger S, Rockstein D, Werlitz V, Pschenitza S, Heidegger P, De Geest S, Valenta S, Teynor A (2021) Implementation science meets software development to create ehealth components for an integrated care model for allogeneic stem cell transplantation facilitated by eHealth: the SMILe study as an example. J Nurs Scholarsh 53(1):35-45. https://doi.org/10.1111/ jnu. 12621

37. Ribaut J, Leppla L, Teynor A, Valenta S, Dobbels F, Zullig LL, De Geest S (2020) Theory-driven development of a medication adherence intervention delivered by eHealth and transplant team in allogeneic stem cell transplantation: the SMILe implementation science project. BMC Health Serv Res 20(1):827. https://doi.org/ 10.1186/s12913-020-05636-1

38. Cohn M (2004) User stories applied: for agile software development. Addison-Wesley Professional

39. Kirsch M, Mitchell SA, Dobbels F, Stussi G, Basch E, Halter JP, De Geest S (2015) Linguistic and content validation of a German-language PRO-CTCAE-based patient-reported outcomes instrument to evaluate the late effect symptom experience after allogeneic hematopoietic stem cell transplantation. Eur J Oncol Nurs 19(1):66-74. https://doi.org/10.1016/j.ejon.2014.07.007

40. Zeiser R, Blazar BR (2017) Pathophysiology of chronic graftversus-host disease and therapeutic targets. N Engl J Med 377(26):2565-2579. https://doi.org/10.1056/NEJMra1703472

41. Howden EJ, La Gerche A, Arthur JF, McMullen JR, Jennings GL, Dunstan DW, Owen N, Avery S, Kingwell BA (2018) Standing up to the cardiometabolic consequences of hematological cancers. Blood Rev 32(5):349-360

42. Hashmi SK, Bredeson C, Duarte RF, Farnia S, Ferrey S, Fitzhugh C, Flowers ME, Gajewski J, Gastineau D, Greenwald M (2017) National institutes of health blood and marrow transplant late 
effects initiative: the Healthcare Delivery Working Group report. Biol Blood Marrow Transplant 23(5):717-725

43. Giunti G, Giunta D, Guisado-Fernandez E, Bender J, FernándezLuque L (2018) A biopsy of breast cancer mobile applications: state of the practice review. Int J Med Inform 110:1-9

44. Collado-Borrell R, Escudero-Vilaplana V, Ribed-Sánchez A, Ibáñez-García S, Herranz-Alonso A, Sanjurjo-Sáez M (2016) Smartphone applications for cancer patients; what we know about them. Farm Hosp 40(1):25-35
45. Zhao J, Freeman B, Li M (2016) Can mobile phone apps influence people's health behavior change? An evidence review. J Med Internet Res 18(11):e287. https://doi.org/10.2196/jmir.5692

Publisher's Note Springer Nature remains neutral with regard to jurisdictional claims in published maps and institutional affiliations. 